Calculating Maximum Entropy Flows in Networks

T. T. Tanyimboh and A. B. Templeman

The Journal of the Operational Research Society

Vol. 44, No. 4, New Research Directions (Apr., 1993), pp. 383-396

This is a post-peer-review, pre-copyedit version of an article published in Journal of the Operational Research Society.

The definitive publisher-authenticated version, i.e. Calculating Maximum Entropy Flows in Networks, T. T. Tanyimboh and A. B. Templeman, The Journal of the Operational Research Society, Vol. 44, No. 4, New Research Directions (Apr., 1993), pp. 383-396, is available online at: http://www.jstor.org/stable/2584416 


\title{
CALCULATING MAXIMUM ENTROPY \\ FLOWS IN NETWORKS
}

\author{
T.T. Tanyimboh and A.B. Templeman \\ Department of Civil Engineering \\ University of Liverpool \\ P.0. Box 147, Liverpool L69 3BX, U.K.
}

\section{ABSTRACT}

The paper describes methods for calculating most likely values of link flows in networks with incomplete data. The object is to present a thorough and rigorous treatment of maximum entropy flow estimation methods and to develop a methodological framework capable of handling different types of network problems. A multiple probability space constrained entropy approach is described for the general network problem. Results are presented and discussed for an example network intended for water supply.

KEY WORDS : networks, information theory, engineering, probability

\section{INTRODUCTION}

The work described in this paper is concerned with methods for probabilistic inference on networks with incomplete data. It was initially stimulated by the problem shown in Figure 1 . Suppose that a pipe network transports water from several source nodes to several demand nodes and that volumetric supplies and demands at all nodes can be measured and are therefore known. Suppose also that the layout of the pipe network and the flow direction in each pipe element are known but no other data whatsoever are available. Under these conditions, how can 'most likely' flow rates in all the pipes of the network be estimated? 
If the layout of pipes in Figure 1 had been a branched system with no loops the supply and demand data would have been sufficient to determine uniquely all the unknown internal flow rates in the pipes. Since the layout in Figure 1 is looped, however, there are more unknowns than equilibrium equations and more information is needed about all the pipes in order to carry out a full looped pipe network analysis. In the absence of this information there are very many possible pipe flow rate distributions which satisfy the equilibrium conditions. Which of these many possible solutions is in some sense most likely and how can it be found?

The problem has practical relevance. Sometimes, especially for old water supply systems, though plan layouts may be available, details of the pipe diameters, friction coefficients and other data may have been lost or may have changed over time. Such water supply networks are usually buried and it may be time consuming and expensive to obtain these data for every pipe element in order to determine the flow rates accurately by calculation. Physical measurements of pipe flow rates requires equipment and can be similarly expensive and time consuming. In these circumstances a method of quickly estimating most likely pipe flow rates would be most useful.

Reduced to its essentials, this problem can be viewed as a transportation problem. There is a set of sources and a set of destinations each supplying or demanding known quantities of flow. Between these two sets of nodes there is some network providing the means of flow transport. It is desired to estimate the most likely internal distribution of flows within this transportation network.

Other problems with these characteristics arise in different practical contexts. For example, consider the well known problem in traffic engineering of estimating the turning flows at a junction or roundabout. Figure $2 \mathrm{a}$ shows a roundabout with four arms, each being a two-way road. Suppose that traffic flow rates can be measured on each arm in each direction thus giving four inflows to and four outflows from the roundabout. On entering the roundabout on any arm each vehicle 
either turns left and leaves by the first exit; or goes straight on and leaves by the second exit, or turns right and leaves by the third exit. The possibility that a driver may make a complete circle and leave by his entrance road is ignored here although this may be included if special circumstances make this significant. For a four arm roundabout and three possible directional choices for a vehicle on any arm, there are therefore twelve unknown turning flows but only seven independent inflow and outflow conditions which they must satisfy. The number of possible solutions is infinite. How can most likely values be estimated for all the twelve unknown turning flows?

Figure $2 \mathrm{~b}$ shows a network representation of the turning flow problem in which the four inflows to the roundabout are shown on the left and the four outflows on the right. Each link of the network represents a possible turning flow whose most likely numerical value must be found. Although the network of Figure $2 b$ is different from that of Figure 1 the nature of the two estimation problems is closely similar in both cases.

The practical problems outlined above, and others, are closely related. Methods already exist ${ }^{1,2,3}$ for solving some of the problems but are incapable of solving more general network problems. The purpose of this paper is to address them in a unified fashion to try to determine a rigorous method capable of tackling all of them. Also there are different interpretations of the term 'most likely' in a flow distribution context. One purpose of this paper is to attempt to clarify that issue. It is shown that the Shannon/Jaynes maximum entropy formalism 4,5 provides both an interpretation of 'most likely' and a methodology for determining most likely flows.

\section{UNCERTAINTY AND NETWORK FLOWS}

The term 'most likely' used with reference to flow rate estimation reflects likelihood in the context of observer uncertainty. In the case of the buried water supply network, when the system is operating and exhibiting the known inflow and outflow rates, the individual pipes have unique water flow rates which obey known physical laws. Although those physical laws are known there is insufficient data to allow the behaviour 
of the system to be calculated from them. The uncertainty in the problem arises not from any randomness or uncertainty in the system itself but from the inability of the observer to deduce its deterministic behaviour. Given sufficient further data, that observer uncertainty could be entirely eliminated and the unique pattern of flows would be revealed. If a method exists whereby some estimate of that flow pattern can be made without extra data, it therefore follows that the method must in some way depend upon and manipulate the observer uncertainty about the physical problem rather than operate methodologically upon the physical processes of the network itself.

The roundabout problem is rather different. Here there are no physical laws governing turning flows. Individual drivers are free to make a choice of turning direction so there is inherent uncertainty in the turning flows themselves. At best, given any amount of extra data, a model of the roundabout system would only be able to estimate some statistical average values for turning flows. Additionally, as with the water supply system, there is observer uncertainty about what any average turning flow rate is. In this paper the 'most likely' estimation process operates upon the observer uncertainty rather than upon the uncertainties in the turning flows themselves.

Figure 3 shows a simplified network which incorporates some, but not all, the characteristic features of the examples described above. In Figure 3 the nature of the flow is not specified; it may be water, vehicles or any unspecified commodity. Let there be $M$ source or supply nodes denoted by $i=1, \ldots, M$ and let $I_{i}$ be the (known) inflow at source node $i$. Let there be $N$ demand or destination nodes denoted by $j=$ $M+1, \ldots, M+N$ and let $O_{M+j}$ be the (known) outflow rate at demand node $j$. Also let there be a total of $L$ direct flow-transporting links $i j$ between source nodes $i$ and demand nodes $j$, and let $t_{i j}$ denote the (unknown) flow rate on link $i j$. Note that the above definitions imply that a node in the network must be either a source or a destination node, there are no other possibilities. Also, the total number of links L may generally be smaller than, and cannot exceed, MN. The network can be described as 
fully-connected if each source node is directly connected to every demand node, in which case the number of links $\mathrm{L}=\mathrm{MN}$. With these definitions the following equations represent flow equilibrium at all nodes of the network.

$$
\begin{aligned}
& \sum_{j=M+1}^{M+N} t_{i j}=I_{i} \quad i=1, \ldots, M \\
& \sum_{i=1}^{M} t_{i j}=0_{j} \quad j=M+1, \ldots, M+N \\
& \text { with } t_{i j} \geq 0 \quad V i j
\end{aligned}
$$

For a balanced problem, in which the sum of all source flows is exactly equal to the sum of all flow demands, Eqs. (1) and (2) are not linearly independent; they contain one redundant equation. Without loss of generality it is assumed that the final demand equation at node $j=$ $M+N$ is omitted. Eqs. (1) and (2) therefore comprise $(M+N-1)$ equations in L unknown link flows. For the purposes of this paper it is assumed that $L>(M+N-1)$, thus a unique solution of Eqs. (1) and (2) does not exist; many different solutions are possible. Values are sought for the L link flows $t_{i j}$ satisfying Eqs. (1) and (2) which are in some sense most likely.

One solution can be found easily if sufficient of the link flows are set to zero until Eqs. (1) and (2) become solveable uniquely for the remaining link flows. This type of result is typically achieved by linear programming if a linear total transportation cost function

$$
\underset{t_{i j}}{\operatorname{Minimize}:} \quad \text { Cost }=\underset{i j}{\sum c_{i j} t_{i j}}
$$

is added to Eqs. (1) and (2). In Eq. (3) $c_{i j}$ represents the cost of transporting one unit of flow along link $i j$. Linear programming simply allocates zero flow along the more expensive links and uses only the cheaper links to carry non-zero flows. However, none of the problems described above is necessarily of this nature; there is no information to 
the effect that cost minimization is involved in them, and no data values for the unit cost coefficients. Consequently there are no grounds for expecting the most likely solution of Eqs. (1) and (2) to have this characteristic pattern.

Indeed, the LP-type solution is a very poor candidate for the title of 'most likely'. Given that possible links exist to carry flow it seems very unlikely that some of them should carry zero flow. The value zero is at one extreme end of the range of possible values for a link flow: it seems intuitively more likely that link flows should have values in the middle of the range rather than at either extreme. Another argument against zero being a most likely value for some link flows comes from the fact that only some, but not all, of the links may have this value. Which links should then be specifically selected to have this zero value rather than any other links? There is no reason to prefer any particular link to have this honour rather than any other link. Extrapolating this argument further, there is no reason to allocate different values to the flow rates in different links unless the equilibrium equations (1) and (2) dictate such a solution. By this reasoning, a most likely set of link flows should be as uniform in value as is permitted by Eqs. (1) and (2).

This characterization of most likely values as most uniform values, subject to satisfying the equilibrium equations, has been developed intuitively. It implicitly uses Laplace's principle of insufficient reason, which requires that in the absence of any good reason to allocate different values to unknown quantities the same value should be allocated to them all. The sufficient reason for a non-uniform choice in this case is that values have to satisfy Eqs. (1) and (2). Laplace's principle therefore leads to the idea that the most likely flows will satisfy Eqs. (1) and (2) and will be as uniform in value as possible.

Laplace's principle is generally recongnized not to be a fundamental principle. It is a consequence of the Shannon/Jaynes maximum entropy formalism 4,5 (MEF) which provides an ideal tool with which to tackle the most likely flow problem of Figure 3. Values must be assigned to the $\mathrm{L}$ link flows and each of those values has some observer uncertainty 
associated with it. If the uncertainty associated with each link flow can be represented probabilistically (or as some probability-like quantity which satisfies all the conditions which are axiomatic to probabilities) then, by virtue of the MEF, the most likely flow rate assignment problem of Figure 3 can be posed as the problem of maximizing the Shannon entropy of the link probabilities subject to whatever is known about the system, i.e. Eqs. (1) and (2).

\section{THE GRAVITY MODEL}

The network flow problems described above are concerned with allocating flow values but the MEF is couched in terms of allocating probabilities. The question of how to introduce probabilities into Figure 3 and Eqs: (1) and (2) is now addressed. Two different ways of doing this will be examined in detail and will be shown to give the same most likely flow values.

The first approach considers the equilibrium equations (1) and (2) and denotes by $\mathrm{T}$ the sum of all link flows in the network. Thus:

$$
T=\sum_{i j} t_{i j}=\sum_{i=1}^{M} I_{i}=\sum_{j=M+1}^{M+N} O_{j}
$$

Probability-like quantities $\mathrm{p}_{i j}$, which satisfy non-negativity and normality conditions associated with probabilities, may be introduced as ratios of link flow $t_{i j}$ to total flow $T$. Thus:

$$
p_{i j}=t_{i j} / T
$$

The most likely flow estimation problem now becomes that of maximizing the Shannon entropy of the link probabilities subject to Eqs. (1) and (2) with link flows substituted by Eq. (5). i.e.

$$
\underset{p_{i j}}{\operatorname{Maximize}:}(\mathrm{S} / \mathrm{K})=-\sum_{i j} \mathrm{p}_{i j} \ln \left(\mathrm{p}_{i j}\right)
$$




$$
\begin{aligned}
& \text { Subject to : } \sum_{i j} p_{i j}=1 \\
& \sum^{\mathrm{M}+\mathrm{N}} \mathrm{p}_{\mathrm{ij}}=\mathrm{I}_{\mathrm{i}} / \mathrm{T} \quad \mathrm{i}=1, \ldots, \mathrm{M} \\
& j=M+1 \\
& \sum_{i=1}^{M} P_{i j}=0_{j} / T \quad j=M+1, \ldots, M+N-1
\end{aligned}
$$

Eq. (6) is the Shannon entropy function ${ }^{4}$ in which $S$ is the entropy and $K$ is an arbitrary positive constant which is not required for maximization. The above problem represented by Eqs. (6) to (9) has a unique solution which may be determined by examining the stationarity of its Lagrangean. The solution may be derived as shown in the Appendix and is:

$$
P_{i j}=I_{i} O_{j} / T^{2} \quad V i j
$$

and Eq. (5) immediately recovers the required estimates of the most likely link flow as:

$$
t_{i j}=I_{i} O_{j} / T \quad V i j
$$

which corresponds to the well-known gravity model of transportation engineering. Substituting the probabilities (10) into the entropy function (6) gives the maximum entropy value for a fully-connected network as:

$$
(\mathrm{S} / \mathrm{K})^{*}=-\sum_{i=1}^{\mathrm{M}}\left(\mathrm{I}_{i} / \mathrm{T}\right) \ln \left(\mathrm{I}_{\mathrm{i}} / \mathrm{T}\right)-\sum_{\mathrm{j}=\mathrm{M}+1}^{\mathrm{M}+\mathrm{N}}\left(\mathrm{O}_{\mathrm{j}} / \mathrm{T}\right) \ln \left(\mathrm{O}_{\mathrm{j}} / \mathrm{T}\right)
$$

In the case of a less-than-fully connected network (such as the roundabout turning flow problem of Figure $2 \mathrm{~b}$ ) result (11) still holds but yields a maximum entropy value $(\mathrm{S} / \mathrm{K})^{*}$ which is smaller than that given by (12). This indicates that in a fully connected network the maximum value 
of the internal entropy is determined by the external macroscopic boundary conditions, but removing links removes potential uncertainty from the internal system and the maximum internal entropy can no longer reach this absolute macroscopic value.

The emergence of the gravity model as representing most likely flow estimates is neither new nor unanticipated. Transportation engineers have been using it for many years as an estimator of traffic flows in a variety of circumstances ${ }^{1}$. Its validity is incontrovertible and is further strengthened by the fact that it may be derived, as here, from first principles as a direct consequence of the maximum entropy formalism. However, it is important to note the restrictions and limitations implicit in Figure 3 and Eqs. (1) and (2). Consequently, the gravity model is directly applicable to the roundabout turning flow problem of Figure 2 but not to the general network problem, for instance the water supply network of Figure 1 in which all links do not start at a source and end at a demand node. Also, in general, there may be several different paths between a source node and a demand node.

In order to handle general networks something more than the gravity model is required. The key to developing an alternative lies in defining probabilities in a different way from Eq. (5), and is described next.

\section{MULTIPLE PROBABILITY SPACE MODELS}

Shannon's entropy is defined for independent and exhaustive probabilities only. These conditions are satisfied by the probabilities in Eq. (5), provided all links of the network start at a source and end at a demand node, as depicted in Figure 3. Essentially, this proviso means that the probabilities in Eqs. (5) are suitable, in the context of entropy, only for networks in which there are no links connected in series. For example, in Figure 1 , all link pairs $(i j, j k), V i, j, k=1, \ldots, N$ are connected in series. In any network with series connections, probability-like terms may still be defined but may not be independent. For any series-connected pair of links ( $i j, j k$ ), the flow in link $j k$ is made 
up, at least in part, of the flow from link ij. As such, these flows are not independent. Consequently, if the corresponding probability-like quantities are defined as in Eqs. (5), those quantities would not be independent.

Refering to Figure 3 again, an alternative way of introducing probabilities is to define $\bar{p}_{i j}$ as the proportion of the total inflow at node $i$ which is carried onwards by link ij (i.e. the probability that an element of the flow which enters node $i$ is transported along link ij to node $j$ ). With this definition, if the inflow at node $i$ is $I_{i}$, then the expected value of the flow on link $i j$ will be $t_{i j}=\bar{p}_{i j} I_{i}$. Substituting this into Eqs. (1) and (2) gives:

$$
\begin{array}{ll}
\sum_{j=M+1}^{M+N} \bar{p}_{i j}=1 & i=1, \ldots, M \\
& \\
\sum_{i=1}^{M} \bar{p}_{i j} I_{i}=O_{j} & j=M+1, \ldots, M+N-1
\end{array}
$$

These equations are equivalent to Eqs. (8) and (9) with the earlier definition of probabilities. Whereas in the earlier formulation there was one normality condition (7) which embraced all the probabilities, there are now $M$ normality conditions (13) and $M$ sets of probabilities. Furthermore these probability sets are not independent: they are conditional upon the probabilities associated with the source node inflows $I_{i}, i=1, \ldots, M$. The question which needs to be addressed is what form of entropy is the correct one to use with multiple dependent probability spaces?

This question has been addressed by Khinchin ${ }^{6}$ who has given several forms of the Shannon entropy function for multiple probability spaces. Two general results are useful:

i) For two independent discrete probability distributions $Q$ and $R$, the entropy of the joint distribution $Q R$ is the sum of the entropies of $Q$ and R separately. Thus: 


$$
S(Q R)=S(Q)+S(R)
$$

ii) For two mutually dependent probability distributions $Q$ and $R$, the entropy of the joint distribution $Q * R$ ( $*$ is used to denote that $Q$ and $R$ are mutually dependent) is given by

$$
S(Q * R)=S(Q)+S(R \mid Q)
$$

where

$$
S(R \mid Q)=\sum_{q} P_{q} S_{q}(R)
$$

$\mathrm{P}_{\mathrm{q}}$ is the probability of the $\mathrm{q}$-th outcome in probability distribution $\mathrm{Q}$, $S_{q}(R)$ is the entropy of probability distribution $R$ conditional upon the $q$-th outcome in probability distribution $Q$ occurring, and $S(R \mid Q)$ is the entropy of probability distribution $R$ conditional upon $Q$ occurring.

Important features of the above results are that $\mathrm{S}(\mathrm{Q} * \mathrm{R})$ is invariant with respect to changes in position of $Q$ and $R$. $S(R * Q)$ is therefore identical to $S(Q * R)$ and is obtained from Eqs. (16) and (17) with the roles of $Q$ and $R$ interchanged. Also $S(Q * R)$ reduces to $S(Q R)$ when $Q$ and $R$ are independent and further reduces to $S(Q)$, the Shannon entropy function (6), when there is only one probability distribution. Extensions to more than two probability distributions follow the rules given above.

Returning to Eqs. (13) and (14), the M probability sets cannot be treated as independent. For a particular set, $i$, probabilities $\bar{p}_{i j}$ measure the likelihood that the inflow at $i$ is transported to demand node $j, j=M+1, \ldots, M+N$. These probabilities within set $i$ are independent but set $i$ itself is conditional upon inflow existing at node $i$. If $p_{i}$ is defined to be the probability of inflow at node $i$ then these probabilities can be determined from the network data: $p_{i}$ is given by the proportion of the total inflows at all source nodes which exists at node i. Thus: 


$$
\mathrm{p}_{i}=\mathrm{I}_{\mathrm{i}} / \sum_{i=1}^{\mathrm{M}} \mathrm{I}_{\mathrm{i}}=\mathrm{I}_{\mathrm{i}} / \mathrm{T} \quad \mathrm{i}=1, \ldots, \mathrm{M}
$$

Eqs. (16) and (17) then give the form of the entropy function to be used with the mutually dependent probability sets defined by Eqs. (13) and (18):

$$
\mathrm{S} / \mathrm{K}=-\sum_{i=1}^{M} \mathrm{p}_{i} \ln \left(\mathrm{p}_{i}\right)-\sum_{i=1}^{M} \mathrm{p}_{\mathrm{i}} \sum_{j=M+1}^{\mathrm{M}+\mathrm{N}} \overline{\mathrm{p}}_{\mathrm{ij}} \ln \left(\overline{\mathrm{p}}_{\mathrm{ij}}\right)
$$

which, on substituting the known probabilities $\mathrm{p}_{i}$ given by Eq. becomes

$$
S / K=-\sum_{i=1}^{M}\left(I_{i} / T\right) \ln \left(I_{i} / T\right)-\sum_{i=1}^{M}\left(I_{i} / T\right) \sum_{j=M+1}^{M+N} \bar{p}_{i j} \ln \left(\bar{p}_{i j}\right)
$$

Most likely flows in the multiple probability space formulation are then given by maximizing the entropy function (20) over probabilities $\bar{p}_{i j}$ for all $i j$, subject to constraints (13) and (14). There is an analytical solution to this problem which can be found by examining the stationarity of its Lagrangean in a similar way to result (10), as given in the Appendix. The solution is:

$$
\overline{\mathrm{p}}_{\mathrm{ij}}=\mathrm{O}_{\mathrm{j}} / \mathrm{T} \quad \mathrm{V} i \mathrm{j}
$$

The link flows are then given by

$$
t_{i j}=\bar{p}_{i j} I_{i}=I_{i} O_{j} / T \quad V i j
$$

which are exactly the same as the flows (11) calculated earlier for the single probability space formulation. Substituting the probabilities (21) into the entropy function (20) and assuming a fully connected network yields the same maximum entropy value (12) given by the earlier formulation.

Two different formulations for determining most likely flows in a network similar to Figure 3 have now been developed. Both the single and 
multiple probability space formulations give identical flow régimes and total uncertainty values. Both formulations are restricted to the conditions associated with Figure 3 and neither is directly applicable to the water supply network of Figure 1 . The next section describes how the multiple probability space formulation can be extended to become applicable to more general networks and uses the Figure 1 example for demonstration purposes.

\section{MULTIPLE PROBABILITY SPACE GENERAL NETWORKS}

Because of difficulties of probabilistic independence, as discussed earlier, the single probability space model cannot easily be extended to a network such as Figure 1. Turning to the multiple probability space approach, the key elements in the model developed earlier for Figure 3 were as follows:

(i) A set of normalized probabilities $\bar{p}_{i j}$ was defined at each node $i$ where the nodal inflow split into at least two outflows. The $\bar{p}_{i j}$ therefore represent the probabilities associated with flow splitting processes.

(ii) The probabilities $\bar{p}_{i j}$ of flows leaving node $i$ were conditional upon probabilities associated with the arrival of inflow at node $i, p_{i}$.

(iii) The entropy function used was the conditional entropy function.

(iv) Constraints upon the entropy function maximization originated in the flow equilibrium equations at all nodes except the final demand node.

These four elements provide the basis for extending the multiple probability space model to more general networks such as that shown in Figure 1. Figure 4 shows the network example of Figure 1 in a more general form with numerical values of source flows and demands replaced by algebraic quantities. Figure $4 \mathrm{a}$ gives details of the node numbering 
and link connectivities, supplies and demands, and also identifies the link flow quantities, $t_{i j}$. Figure $4 \mathrm{~b}$ gives details of all the probabilities associated with this particular problem. First, all the elements of the model are assembled according to requirements (i) to (iv) above.

In accordance with (i) there are flow splitting processes at the source nodes 1 and 2 , and also at nodes 3,4 and 5 . At the source nodes probabilities are easily defined as ratios of link outflows to the total link outflows at each node. Thus, since the total of the link outflows is in this case equal to the known source inflow:

$$
\begin{array}{ll}
\overline{\mathrm{p}}_{13}=t_{13} / \mathrm{I}_{1} \quad ; \quad \overline{\mathrm{p}}_{14}=t_{14} / \mathrm{I}_{1} \\
\overline{\mathrm{p}}_{23}=\mathrm{t}_{23} / \mathrm{I}_{2} \quad ; \quad \overline{\mathrm{p}}_{24}=t_{24} / \mathrm{I}_{2}
\end{array}
$$

The flow splitting probabilities at nodes 3,4 and 5 are also ratios of individual outflows to the sum of the outflows at a node and lead to the following definitions of probabilities. Note particularly that a probability must be assigned to the demand from a node. The reason for doing this is that, although the demand at a node is known, the total outflow from a node is not known. Hence the ratio of the demand to the total outflow is not known and a probability is required to represent this. The use of zero as a second suffix denotes these demand probabilities. Thus:

$$
\overline{\mathrm{p}}_{34}=\mathrm{t}_{34} / \mathrm{T}_{3} \quad ; \quad \overline{\mathrm{p}}_{35}=\mathrm{t}_{35} / \mathrm{T}_{3} \quad ; \quad \overline{\mathrm{p}}_{30}=0_{3} / \mathrm{T}_{3}
$$

in which $T_{3}=t_{34}+t_{35}+0_{3}$,

$$
\overline{\mathrm{p}}_{46}=\mathrm{t}_{46} / \mathrm{T}_{4} \quad ; \quad \overline{\mathrm{p}}_{40}=\mathrm{O}_{4} / \mathrm{T}_{4}
$$

with $\mathrm{T}_{4}=\mathrm{t}_{46}+\mathrm{O}_{4}$, and

$$
\overline{\mathrm{p}}_{56}=t_{56} / \mathrm{T}_{5} \quad ; \quad \overline{\mathrm{p}}_{50}=0_{5} / \mathrm{T}_{5}
$$

with $\mathrm{T}_{5}=\mathrm{t}_{56}+\mathrm{O}_{5}$.

The way in which Eqs. (22) define the probability sets ensures that they satisfy normality without the need for separate normality constraints. 
Non-negativity of the probabilities is also ensured provided that link flows are always in the direction defined and never become negative.

In accordance with (ii), all the above probabilities are conditional upon flow existing at the nodes at which these flow splitting probabilities are defined. In the case of the source nodes 1 and 2 the probabilities that flow exists there are known and are given by the first equality in Eq. (18).

$$
p_{1}=I_{1} /\left(I_{1}+I_{2}\right) \quad ; \quad p_{2}=I_{2} /\left(I_{1}+I_{2}\right)
$$

In accordance with (iii) the conditional entropy function for the network of Figure 4 may be assembled systematically using the principles of conditional probability and the conditional entropy definitions (16) and (17). Starting with the two source nodes there is the entropy, $S_{0}$, of the probabilities $p_{1}$ and $p_{2}$ that flow exists at the source nodes. This is :

$$
S_{0}=-p_{1} \ln \left(p_{1}\right)-p_{2} \ln \left(p_{2}\right)
$$

There are then the entropies of the probabilities associated with the link flows leaving the two source nodes. For node 1 , probabilities $\overline{\mathrm{p}}_{13}$ and $\bar{p}_{14}$ are conditional upon $\mathrm{p}_{1}$, and the conditional entropy, $\mathrm{s}_{1}$, is therefore:

$$
\mathrm{s}_{1}=-\mathrm{p}_{1}\left[\overline{\mathrm{p}}_{13} \ln \left(\overline{\mathrm{p}}_{13}\right)+\overline{\mathrm{p}}_{14} \ln \left(\overline{\mathrm{p}}_{14}\right)\right]
$$

For node 2 , probabilities $\overline{\mathrm{p}}_{23}$ and $\overline{\mathrm{p}}_{24}$ are conditional upon $\mathrm{p}_{2}$ and the entropy is therefore:

$$
\mathrm{S}_{2}=-\mathrm{p}_{2}\left[\overline{\mathrm{p}}_{23} \ln \left(\overline{\mathrm{p}}_{23}\right)+\overline{\mathrm{p}}_{24} \ln \left(\overline{\mathrm{p}}_{24}\right)\right]
$$

At node 3 there is entropy associated with the probabilities for link flows leaving node 3 . These flows are conditional upon flow arriving at node 3. Flow can arrive at node three by two routes: from node 1 via link 1-3, and from node 2 via link 2-3. The probability of flow arriving at node 3 by the first of these routes is $p_{1} \bar{p}_{13}$ and by the second route is $p_{2} \bar{p}_{23}$. Thus the conditional entropy at node 3 is: 
$S_{3}=-\left[\bar{p}_{1} \bar{p}_{13}+p_{2} \bar{p}_{23}\right]\left[\bar{p}_{34} \ln \left(\overline{\mathrm{p}}_{34}\right)+\bar{p}_{35} \ln \left(\overline{\mathrm{p}}_{35}\right)+\overline{\mathrm{p}}_{30} \ln \left(\overline{\mathrm{p}}_{30}\right)\right]$

Similarly for node 4 the entropy of the flows leaving node 4 is conditional upon flow arriving at node 4 from the source nodes 1 and 2 , and also from node 3 . Thus the conditional entropy at node 4 is:

$$
\begin{aligned}
\mathrm{S}_{4}=-\left[\mathrm{p}_{1} \overline{\mathrm{p}}_{14}\right. & \left.+\mathrm{p}_{2} \overline{\mathrm{p}}_{24}+\overline{\mathrm{p}}_{34}\left(\mathrm{p}_{1} \overline{\mathrm{p}}_{13}+\mathrm{p}_{2} \overline{\mathrm{p}}_{23}\right\}\right]\left[\overline{\mathrm{p}}_{46} \ln \left(\overline{\mathrm{p}}_{46}\right)\right. \\
& \left.+\overline{\mathrm{p}}_{40} \ln \left(\overline{\mathrm{p}}_{40}\right)\right]
\end{aligned}
$$

Similarly at node 5 the conditional entropy turns out to be:

$\mathrm{S}_{5}=-\left[\overline{\mathrm{p}}_{35}\left\{\mathrm{p}_{1} \overline{\mathrm{p}}_{13}+\mathrm{p}_{2} \overline{\mathrm{p}}_{23}\right\}\right]\left[\overline{\mathrm{p}}_{56} \ln \left(\overline{\mathrm{p}}_{56}\right)+\overline{\mathrm{p}}_{50} \ln \left(\overline{\mathrm{p}}_{50}\right)\right]$

The conditional entropy of the entire network of Figure 4 is then simply the sum of the separate entropies in Eqs. (24a) to (24f):

$$
\mathrm{S} / \mathrm{K}=\mathrm{S}_{0}+\mathrm{S}_{1}+\mathrm{S}_{2}+\mathrm{S}_{3}+\mathrm{S}_{4}+\mathrm{S}_{5}
$$

In accordance with (iv), constraints are generated by the flow equilibrium conditions at all nodes except the final demand node, node 6 in the network of Figure 4, and are:

$$
\begin{aligned}
& t_{13}+t_{14}=I_{1} \\
& t_{23}+t_{24}=I_{2} \\
& t_{13}+t_{23}=t_{34}+t_{35}+o_{3} \\
& t_{14}+t_{24}+t_{34}=t_{46}+o_{4} \\
& t_{35}=t_{56}+o_{5}
\end{aligned}
$$

The above model contains both link flow unknowns $t_{i j}$ and probability unknowns $\dot{\mathrm{p}}_{\mathrm{ij}}$. which are connected through the probability definitions (22). The first step in solving the model consists of simplifying it to an easily solveable form. There are several ways in which this can be done; the way chosen here is to express everything in terms of a reduced number of link flow variables. The five flow equilibrium constraints (26) contain eight link flow unknowns but are equalities. They can therefore be used to express all link flows in terms of just three independent link flow variables. Choosing $t_{14}, t_{24}$ and $t_{34}$ as the independent variables yields: 


$$
\begin{array}{rrrl}
t_{13} & = & I_{1}-t_{14} & \\
t_{23} & = & I_{2} & -t_{24} \\
t_{35} & = & I_{1}+I_{2}-o_{3}-t_{14}-t_{24}-t_{34} \\
t_{46}= & -o_{4}+t_{14}+t_{24}+t_{34} \\
t_{56}=I_{1}+I_{2}-o_{3}-o_{5}-t_{14}-t_{24}-t_{34}
\end{array}
$$

Definitions (22a) to (22e) may then be used to express all the probabilities $\bar{p}_{i j}$ in terms of unknowns $t_{14}, t_{24}$ and $t_{34}$, and the entropy function (25) then becomes a function only of these three variables.

The flow directions defined in Figure $4 \mathrm{a}$ require that all the link flow quantities $t_{i j}$ should be non-negative. The final model should therefore consist of maximizing the entropy function (25) over the link flow variables $t_{14}, t_{24}$ and $t_{34}$ subject to constraints that all the right-hand side functions in Eqs. (27) and the link flow variables themselves must be non-negative. However, the final model was actually solved by NAG library routine E04JAF which is an unconstrained optimization routine, and the constraints were omitted whilst maximizing the entropy function. The optimum solution was then substituted into the constraints to check that they were all satisfied but inactive. The reasons for adopting this approach were that it provides a much simpler and quicker solution, and that, as has been argued earlier, it is expected that the maximum entropy solution will have flows which are as uniform as possible without any being equal to zero.

The entropy function '25) for the example of Figure 4 was constructed somewhat laboriously using the conditional entropy definitions (16) and (17). In fact its form is quite simple and has only two types of terms. The first type comprises the entropy of the source flow probabilities, $\mathrm{S}_{0}$, given by Eq. (24a). In most network examples the source flows are known and form part of the available data, so these source flow entropy terms are actually known constants. They may be omitted from the objective function maximization and are not necessary to the solution process. The only case in which they are needed is that of a network in which source flows are not specified and must be estimated along with the pipe flows. The form of this first type of terms is always 


$$
\mathrm{S}_{0}=-\sum_{i=1}^{\mathrm{M}} \mathrm{p}_{\mathrm{i}} \ln \left(\mathrm{p}_{\mathrm{i}}\right) \quad \mathrm{i}=1, \ldots, \mathrm{M}
$$

in which $p_{i}$ is as defined in Eq. (18).

The second type of terms are $S_{1}$ to $S_{5}$ given by Eqs. (24b) to (24f). Each of these equations represents the conditional entropy at a node of the network where flow splitting probabilities are defined. The form of these terms is always the same and consists of the entropies of all the outflow probabilities, including that of any demand at the node, multiplied by the total probability of flow arriving at that node by all possible paths. The form of these terms at any node $k$ in the network is therefore

$$
\left.S_{k}=-p_{k} \underset{k j \in N_{k}}{\left[\sum\right.} \bar{p}_{k j} \ln \left(\bar{p}_{k j}\right)\right]
$$

in which $\mathrm{N}_{\mathrm{k}}$ is the subset of outflows from node $\mathrm{k}$ including any demand. $p_{k}$ is the total probability of flow arriving at node $k$ by all possible paths.

All nodes of the network generate terms of the form of Eq. (29) and the entropy function sums them all. At a node which has only one outflow (either a demand or a link outflow) the single entropy term in the [ ] in Eq. (29) is zero by virtue of the way in which probabilities $\bar{p}_{k j}$ are defined. This accords with intuition since at such a node there should be no additional uncertainty to what already exists elsewhere in the network. The entropy function as defined by Eqs. (28) and (29) therefore has a conveniently structured form which permits it to be assembled easily for any network.

The network of Figure 1 is used as a numerical example of the calculation of maximum entropy flows in a general network. Table 1 gives numerical results for two instances of Figure 1 . In case $A$ the inflows and outflows are exactly as shown in Figure 1 . In case $B$ the inflows at nodes 1 and 2 are interchanged. In both cases the link flow directions are as in Figure 1. 


\section{DISCUSSION}

The results in Table 1 show several interesting features. Firstly, the link flows in the lower half of the network are the same for both cases $A$ and B. Further study reveals that they remain the same for any combination of source flows at nodes 1 and 2 totalling 55 units. The reason for this is that nodes 1 and 2 are each connected to the rest of the network in exactly the same fashion, and they provide flow to nodes 3 and 4 in exactly the same proportions. From Table 1 the proportions of source flow transmitted from each source to nodes 3 and 4 may be calculated as 0.7121 and 0.2879 respectively. These proportions of the total available flow are required at nodes 3 and 4 in order to serve the demands in the rest of the network using maximum entropy pipe flows. The network is unable to distinguish topologically between the two source nodes, and, for demands as in Figure 1, any combination of source flows at nodes 1 and 2 which totals 55 units will be distributed to nodes 3 and 4 in these same proportions.

Secondly, a means of partially checking the validity of the results of Table 1 is to consider the example of Figure 1 with all directions reversed. The two source flows become demands, the four demands become sources, and the flow directions along all links are reversed. A complete reversal of all directions in this fashion completely changes the definitions of flow splitting probabilities and leads to a different conditional entropy function from Eq. (25). However, it should not change the intrinsic total uncertainty (entropy) in the system. Solving the reversed model, therefore, should yield exactly the same results as those of Table 1 . It does, and this provides reassurance that the calculation are correct.

A third comment which may be made on the results of Table 1 is that the invariance of the most likely flow values in the links in the lower half of the network is reassuring from both design and reliability viewpoints. In the case of a water supply network, designing the pipes to carry these flows would appear to confer a considerable degree of 
invulnerability upon the lower half of the network to possible variations in the source flows. The issue of network reliability and the importance of maximum entropy flows in this context is too large for detailed examination here but has been studied by Awumah, Goulter and Bhatt ${ }^{2,3}$.

\section{CONCLUSIONS}

The problem of estimating from incomplete data most likely values of flows on the links of a general network has been studied. Most likely flows were first characterized as those flows which maximize an entropy function for the network subject to the available information. Possible forms of this entropy function were examined and it was shown that a multiple probability space conditional entropy model was most appropriate for general network problems. The detailed solution of a typical general network problem was presented and the results discussed.

A considerable amount of work still needs to be done. The paper has established the basic elements and outlined the structure of a computer method for the calculation of most likely flows in any general network. That computer program needs to be written and tested and the results examined. The nature of these maximum entropy flows needs to be studied and interpreted. Conjectures about their potential value in the design of engineering networks and about possible close relationships between entropy and reliability need to be critically-examined and tested. The ability to infer most likely values in networks has many potential uses which need to be explored. Essentially, this paper has shown how the calculations may be done: the value of the results remains to be established.

\section{REFERENCES}

1. S. Erlander (1977) Accessibility, entropy and the distribution and assignment of traffic. Transportation Research, 11, 149-153.

2. K. Awumah, I. Goulter and S.K. Bhatt (1990) Assessment of reliability in water distribution networks using entropy based measures. Stochastic Hydrology and Hydraulics, 4, (4), 325-336. 
3. K. Awumah, I. Goulter and S.K. Bhatt (1991) Entropy-based redundancy measures in water distribution network design. ASCE J. Hydraulic Engineering, 117, (5), 595-614.

4. C.E. Shannon (1948) A mathematical theory of communication. Bell System Technical J., 27, (3), 379-428.

5. E.T. Jaynes (1957) Information theory and statistical mechanics. Phys. Rev., 106, 620-630 and 108, 171-190.

6. A.I. Khinchin (1953) The entropy concept in probability theory. Uspekhi Matematicheskikh Nauk, 8, (3), 3-20. Translation in A.I. Khinchin (1957) Mathematical Foundations of Information Theory, Dover, New York pp. 1-28. 
APPENDIX Derivation of result (10)

The Lagrangean of protlem (6)-(9) may be written as:

$$
\begin{aligned}
& L(\underset{\sim}{p}, \lambda, \underset{\sim}{\alpha}, \underset{\sim}{\beta})=-\sum_{i j} p_{i j} \ln \left(p_{i j}\right)+(1+\lambda)\left(\begin{array}{ll}
\sum_{i j} & p_{i j}-1
\end{array}\right) \\
& +\sum_{i=1}^{M} \alpha_{i}\left(\sum_{J=M+1}^{M+N} p_{i j}-I_{i} / T\right)+\sum_{j=M+1}^{M+N-1} \beta_{j}\left(\sum_{i=1}^{M} p_{i j}-0_{j} / T\right)
\end{aligned}
$$

Stationarity of the Lagrangean with respect to a typical probability, $P_{i}, j$, yields

$$
\begin{array}{r}
-\ln \left(\mathrm{p}_{i, j},\right)-1+(1+\lambda)+\alpha_{i},+\beta_{j},=0 \\
\mathrm{p}_{i, j},=\exp (\lambda) \exp \left(\alpha_{i},+\beta_{j},\right)
\end{array}
$$

Stationarity of the Lagrangean with respect to $\lambda$ yield the normality condition (7). Substituting (A1) in (7) gives

$$
\begin{aligned}
\exp (\lambda) \sum_{i j} \exp \left(\alpha_{i}+\beta_{j}\right)=1 \\
\\
\exp (\lambda)=1 / \sum_{i j} \exp \left(\alpha_{i}+\beta_{j}\right)
\end{aligned}
$$

Substituting (A2) in (A1) gives

$$
\begin{aligned}
& \mathrm{p}_{\mathrm{i}, j},=\exp \left(\alpha_{\mathrm{i}},+\beta_{\mathrm{j}},\right) / \sum_{i j} \exp \left(\alpha_{i}+\beta_{j}\right)
\end{aligned}
$$

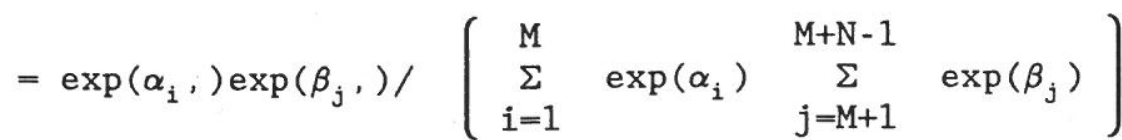

Stationarity of the Lagrangean with respect to a typical multiplier, $\alpha_{i}$, , yields the inflow constraint ( 8 ) for node $i^{\prime}$ :

$$
\sum_{j=M+1}^{M+N} P_{i}, j=I_{i}, / T
$$


Substituting result (A3), this becomes

$$
\begin{gathered}
\exp \left(\alpha_{i},\right) \underset{j=M+1}{M+N-1} \exp \left(\beta_{j},\right) /\left(\sum_{i=1}^{M} \exp \left(\alpha_{i}\right) \sum_{j=M+1}^{M+N-1} \exp \left(\beta_{j}\right)\right) \\
=I_{i}, / T \\
\exp \left(\alpha_{i},\right) / \sum_{i=1}^{M} \exp \left(\alpha_{i}\right)=I_{i}, / T
\end{gathered}
$$

Stationarity of the Lagrangean with respect to a typical multiplier, $\beta_{j}$, yields the outflow constraint (9) for node $j^{\prime}$ :

$$
\sum_{i=1}^{M} p_{i j},=0_{j}, / T
$$

Substituting result (A3) and proceeding as for $\alpha_{i}$, this gives

$$
\exp \left(\beta_{j},\right) / \sum_{j=M+1}^{M+N-1} \exp \left(\beta_{j}\right)=0_{j} / T
$$

Substituting results (A4) and (A5) into (A3) gives

$$
\begin{aligned}
p_{i}, j & =\left[\exp \left(\alpha_{i},\right) / \sum_{i=1}^{M} \exp \left(\alpha_{i}\right)\right]\left[\exp \left(\beta_{j},\right) / \sum_{j=M+1}^{M+N-1} \exp \left(\beta_{j}\right)\right] \\
& =\left[I_{i}, / T\right]\left[0_{j}, / T\right] \\
p_{i, j}, & =I_{i}, O_{j}, / T^{2}
\end{aligned}
$$

which is result (10) when generalized for all $i$ and $j$.

Result (21) for the multiple probability space model may be derived in a closely similar manner by examining the Lagrangean of the problem defined by Eqs. (20), (13), and (14). 
Table 1 Maximum entropy flows for the network of Figure 1

\begin{tabular}{rrr}
\hline & \multicolumn{2}{c}{ flow rate } \\
\cline { 3 - 3 } link & & \\
& & \\
& & case B \\
& & \\
$1-3$ & & \\
$1-4$ & 14.242 & 24.924 \\
$2-3$ & 5.758 & 10.076 \\
$2-4$ & 24.924 & 14.242 \\
$3-4$ & 10.076 & 15.838 \\
$3-5$ & 15.833 & 8.333 \\
$4-6$ & 8.333 & 6.667 \\
$5-6$ & 6.667 & 3.333 \\
\hline$(S / K)$ & 3.333 & 2.41098 \\
\hline
\end{tabular}




\section{FIGURE CAPTIONS}

Figure 1 Water supply network

Figure 2 a) Four-arm roundabout, and b) the turning flows represented as links of a network

Figure 3 Network notation

Figure 4 Water supply network example of Figure 1

a) Supply, demand and flow definitions

b) Probabilities

\section{TABLE CAPTION}

Table 1 Maximum entropy flows for the network of Figure 1 


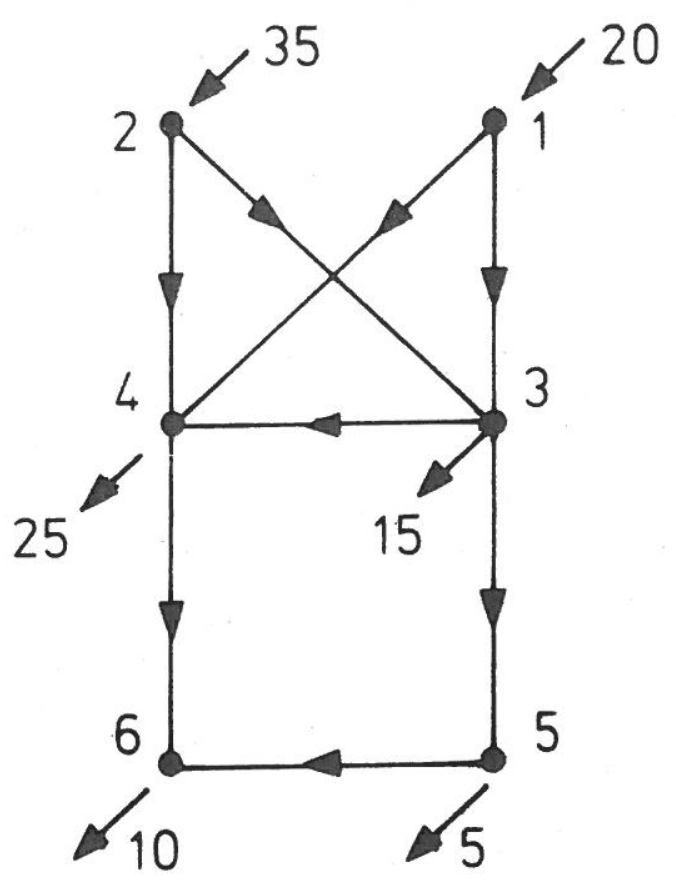

Fig. 1

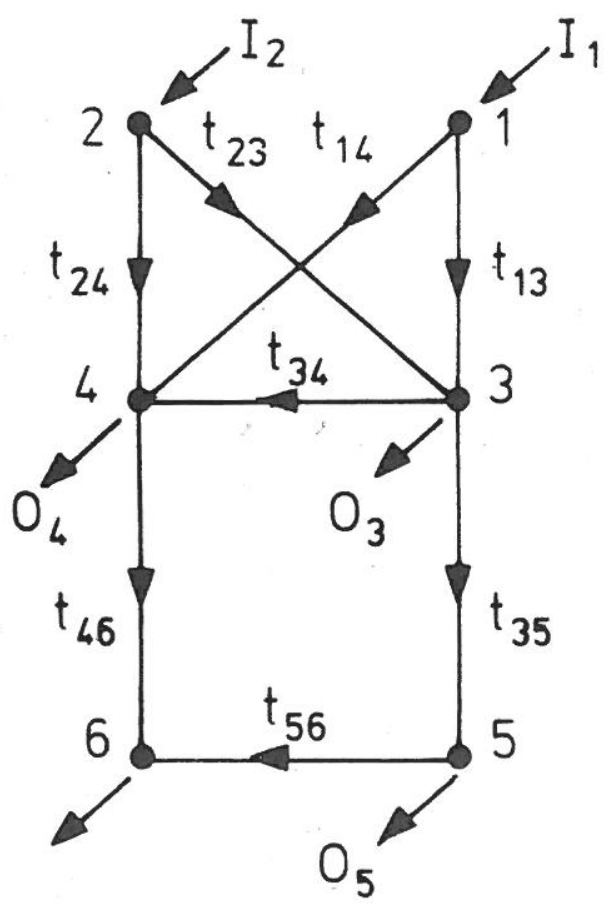

(a)

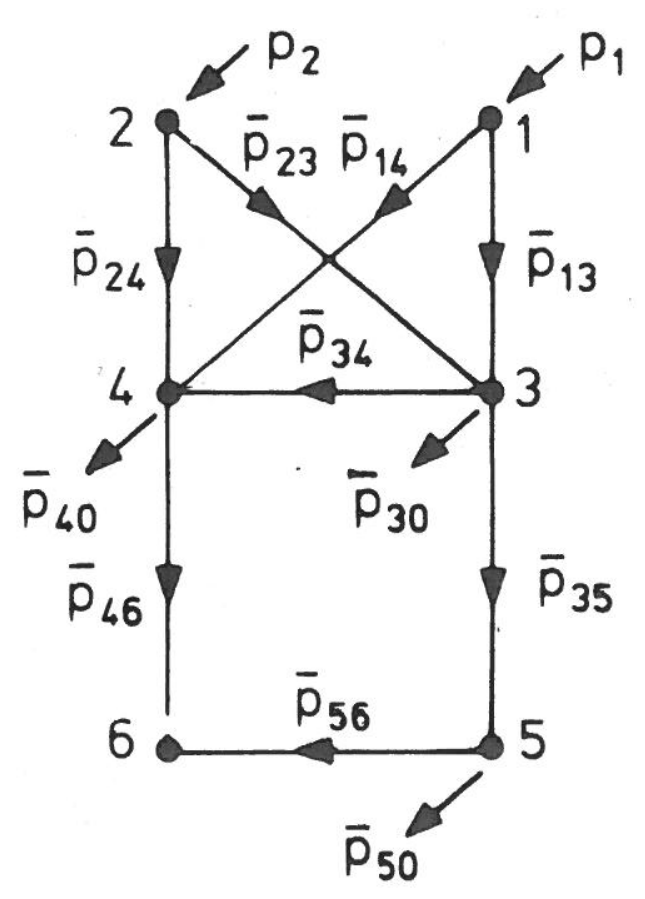

(b)

Fig. 4 

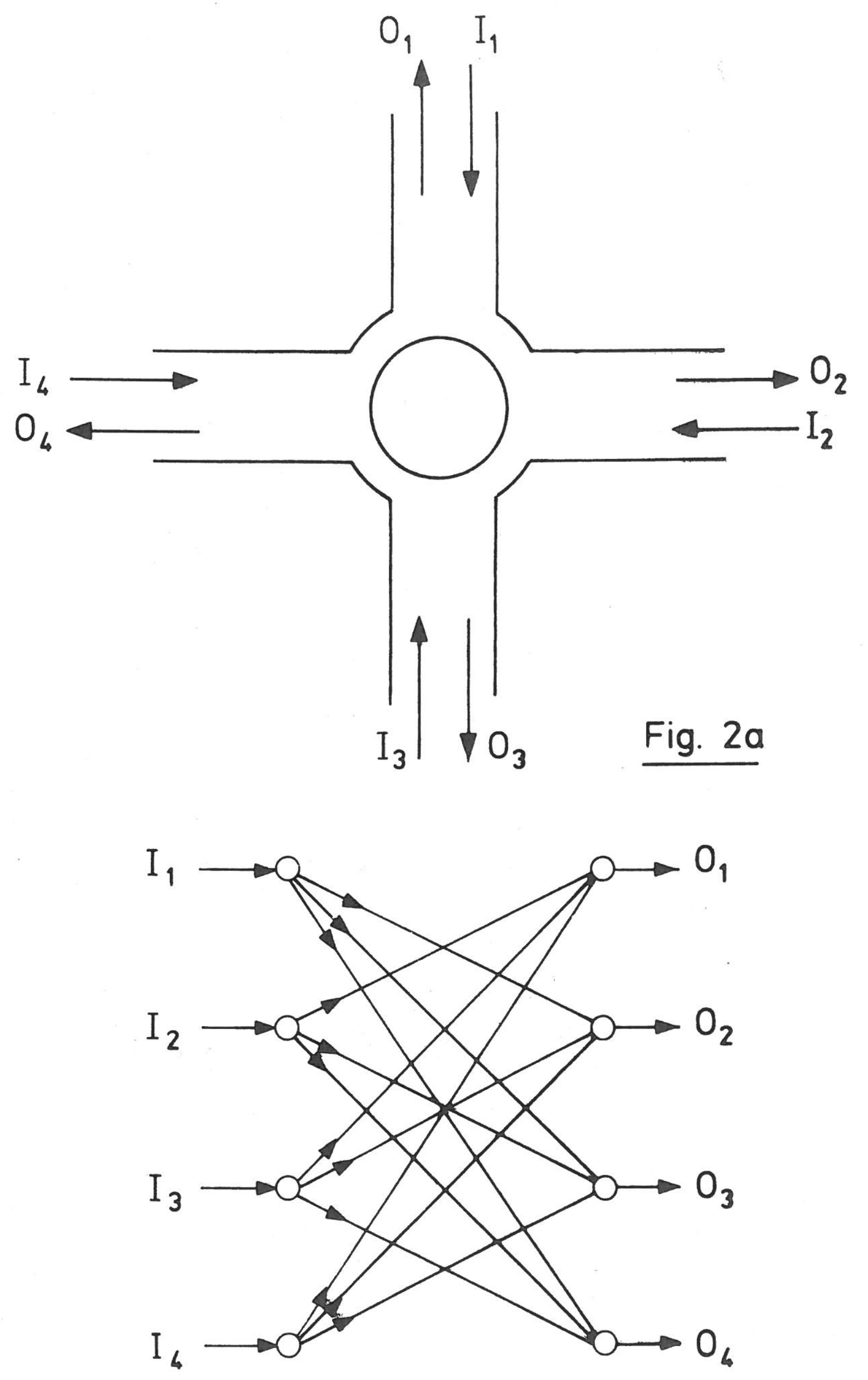

Fig. 2b 


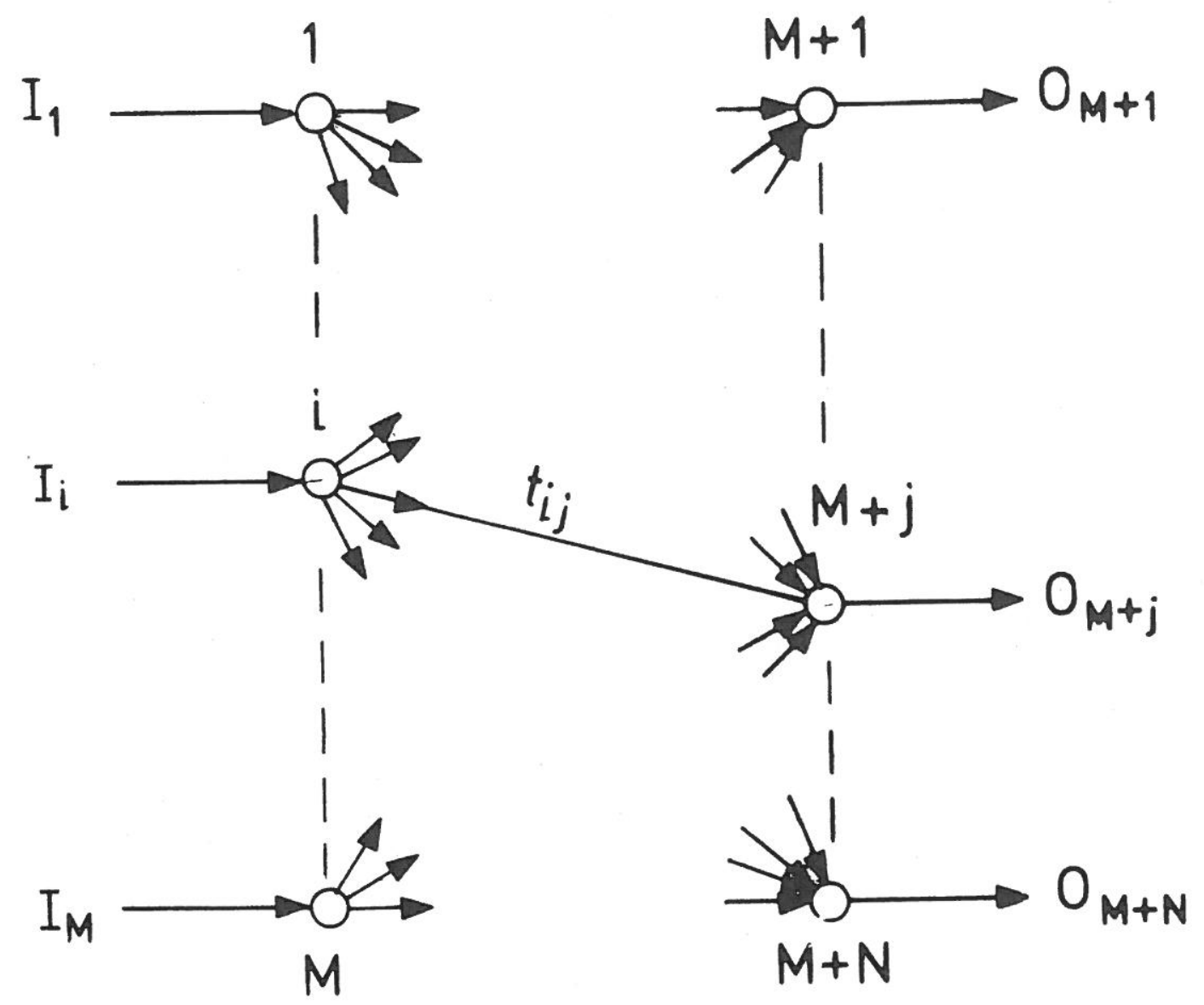

Fig. 3 